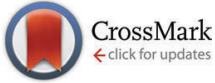

Cite this: Chem. Commun., 2015, 51, 15788

Received 24th June 2015,

Accepted 31st August 2015

DOI: $10.1039 / c 5 c c 05209 b$

www.rsc.org/chemcomm

\section{Dual metal and Lewis base catalysis approach for asymmetric synthesis of dihydroquinolines and the $\alpha$-arylation of aldehydes via $N$-acyliminium ions $\uparrow$}

\author{
Chandra M. R. Volla, ${ }^{a}$ Eleonora Fava, ${ }^{b}$ Iuliana Atodirese ${ }^{\mathrm{b}}$ and Magnus Rueping ${ }^{\star b}$
}

\begin{abstract}
A dual catalytic system consisting of indium triflate and a chiral imidazolidinone catalyzes the asymmetric addition of aldehydes to $\mathrm{N}$-acyl quinoliniums furnishing optically active dihydroquinolines in good yields and excellent selectivities. The products were further functionalized into optically active tetrahydroquinolines, quinolines and 6-oxa-2-aza-bicyclo[3.3.1]nonanes.
\end{abstract}

Nitrogen-containing heterocycles constitute common frameworks present in drugs and biologically active molecules and are therefore important classes of compounds for pharmaceutical and agricultural industries. In particular the quinoline unit is a prevalent structural motif found in a wide range of natural products and biologically active substances. ${ }^{1}$ Despite its high significance, only a limited number of asymmetric methods were developed for the enantioselective synthesis of this class of compounds. ${ }^{2}$ For example, acylation of nitrogen containing heteroaromatics like quinolines makes them highly electrophilic and hence they undergo addition reactions with a variety of nucleophiles. In 2000 Shibasaki reported the first catalytic, enantioselective Reissert-type addition ${ }^{3}$ of trimethylsilylcyanide to quinolines using bifunctional BINOL-derived catalysts. ${ }^{4}$ Chiral thiourea catalysts having a pendant hydroxyl were engaged by Takemoto and co-workers for the asymmetric Petasis type addition of boronic acids to quinolines. ${ }^{5,6}$ These methods involve in situ formation of active $N$-acyl iminium species by the reaction of heteroaromatics and chloroformate. In contrast Doyle and co-workers reported an enantioselective nickel-catalyzed addition of boronates to stable 2-ethoxy-1-ethoxycarbonyl-1,2-dihydroquinolines (EEDQ). ${ }^{7}$ More recently, Schaus and co-workers developed an asymmetric addition of vinylboronic acids to EEDQs using simple and inexpensive tartaric acid as catalyst. ${ }^{8}$ Given the easy availability of EEDQs we envisioned their use as electrophiles in asymmetric

\footnotetext{
${ }^{a}$ Department of Chemistry, Indian Institute of Technology Bombay, Powai, Mumbai, 400076, Maharashtra, India

${ }^{b}$ Institute of Organic Chemistry, RWTH Aachen University, Landoltweg 1, 52074, Aachen, Germany. E-mail: magnus.rueping@rwth-aachen.de

$\dagger$ Electronic supplementary information (ESI) available. See DOI: 10.1039/ c5cc05209b
}

reactions with aldehydes, as this protocol would allow the expeditious asymmetric synthesis of chiral quinoline derivatives.

The activation of aldehydes by secondary amines to form enamines and iminium ions has revolutionized the field of organocatalysis. ${ }^{9}$ In particular, the enamine catalysis working on the principle of HOMO activation has been exploited in reactions with a wide range of electrophiles including acyliminium ions derived from hetero-aromatics. For example, chiral pyrrolidine derivatives were used for the intramolecular addition of aldehydes onto isoquinolinium ions resulting in optically active 1,2-dihydroisoquinoline derivatives. ${ }^{10}$ An intermolecular version of this transformation was also reported recently. ${ }^{11}$ Furthermore, the use of copper salts together with chiral prolinol ethers in the enantioselective, oxidative cross-dehydrogenative coupling of $\mathrm{N}$-aryl tetrahydroisoquinolines with aldehydes was reported. ${ }^{12,13}$ Despite these advances, the enantioselective synthesis of dihydroquinolines using similar strategies was not reported to date.

Herein we describe the asymmetric addition of aldehydes to quinoline acetals to accomplish the efficient asymmetric synthesis of chiral quinolines (Scheme 1). ${ }^{14}$

In order to circumvent potential problems related to the sensitivity of the iminium ion intermediate and the generation of stoichiometric amounts of acid byproduct, we envisioned performing asymmetric enamine catalysis with EEDQ, a stable precursor of quinolinium ion that will generate only non-toxic, non-corrosive by products and apply either a Brønsted or Lewis acid catalyst to activate the quinoline acetal. We tested our

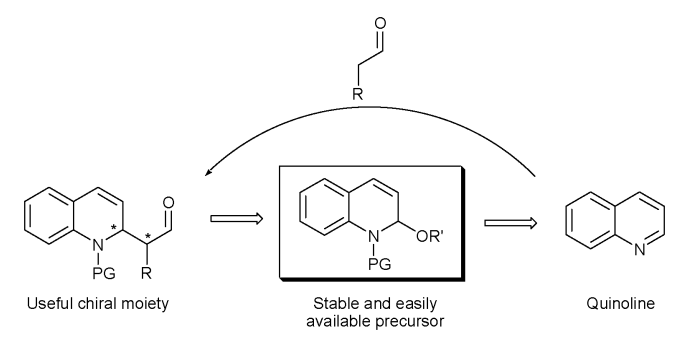

Scheme 1 Asymmetric addition of aldehydes to quinoline acetals. 
Table 1 Optimization of reaction conditions for the addition of propanal to quinoline acetal

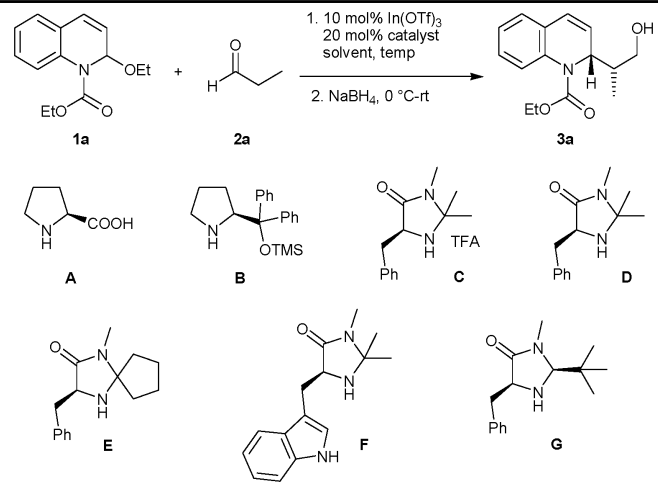

\begin{tabular}{|c|c|c|c|c|c|c|c|}
\hline Entry & Cat. & Solvent & $T\left({ }^{\circ} \mathrm{C}\right)$ & Time (h) & Yield $^{a}(\%)$ & d.r. ${ }^{b}$ & $\mathrm{ee}^{c}(\%)$ \\
\hline 1 & A & DCM & $\mathrm{RT}$ & 12 & 62 & $1: 1$ & -11 \\
\hline 2 & B & DCM & RT & 12 & 74 & $1: 1$ & 38 \\
\hline 3 & C & DCM & RT & 6 & 84 & $1: 1$ & 19 \\
\hline 4 & - & DCM & $\mathrm{RT}$ & 12 & - & - & - \\
\hline 5 & $\mathbf{C}^{d}$ & DCM & RT & 24 & - & - & - \\
\hline 6 & D & DCM & RT & 12 & 73 & $1: 1$ & 63 \\
\hline 7 & $\mathbf{E}$ & DCM & RT & 12 & 72 & $1: 1$ & 57 \\
\hline 8 & $\mathbf{F}$ & DCM & RT & 12 & 67 & $1: 1$ & 54 \\
\hline 9 & D & DCM & 0 & 18 & 72 & $1.8: 1$ & 87 \\
\hline 10 & G & DCM & 0 & 18 & 69 & $1: 1$ & $91^{e}$ \\
\hline 11 & D & $\mathrm{CHCl}_{3}$ & 0 & 18 & 75 & $2: 1$ & 92 \\
\hline 12 & D & Toluene & 0 & 18 & 78 & $3: 1$ & 94 \\
\hline 13 & D & $\mathrm{CH}_{3} \mathrm{CN}$ & 0 & 18 & 62 & $1.5: 1$ & 47 \\
\hline 14 & D & THF & 0 & 18 & 68 & $2.3: 1$ & 92 \\
\hline 15 & D & Ethanol & 0 & 18 & 56 & $2: 1$ & 89 \\
\hline $16^{f}$ & D & Toluene & 0 & 24 & 72 & $4: 1$ & 93 \\
\hline $17^{f}$ & D & Toluene & -10 & 24 & 69 & $4: 1$ & 96 \\
\hline
\end{tabular}

${ }^{a}$ Yield after column chromatography. ${ }^{b}$ Diastereomeric ratio was determined by ${ }^{1} \mathrm{H}$-NMR. ${ }^{c}$ Enantiomeric excess of the major diastereomer was determined by chiral HPLC analysis. The enantiomeric excess of the minor diastereomer is given in the ESI. ${ }^{d}$ Reaction was done in the absence of $\operatorname{In}(\mathrm{OTf})_{3} \cdot{ }^{e}$ Enantiomeric excess of minor diastereomer. ${ }^{f}$ Reaction was done using i-butyl carbamate instead of ethyl carbamate.

hypothesis using quinoline acetal 1a and propionaldehyde (2a) as reaction partners. Different organocatalysts were evaluated in the model reaction using $10 \mathrm{~mol} \%$ of indium triflate as Lewis acid. ${ }^{15-16}$ The products were reduced with sodium borohydride after reaction for a facile analysis (Table 1). While proline (A) gave the product $3 \mathrm{a}$ with $62 \%$ yield and $11 \%$ ee in DCM solvent, TMS-protected diphenylprolinol ether (B) afforded the product in higher yield and selectivity (Table 1, entries 1 and 2).

When imidazolidinone TFA salt $\mathbf{C}$ was used as catalyst, the reaction was faster and gave the product in $84 \%$ yield and 19\% ee (Table 1, entry 3). Both Lewis acid and chiral secondary amine are playing key roles as in the absence of either of them no reaction was observed (Table 1 , entries 4 and 5). This also demonstrates that an effective interplay between the two catalysts is necessary for the efficient creation of stereogenic centers. We were delighted to see that using the free amine $\mathbf{D}$ instead of the corresponding salt had a dramatic effect on the selectivity of the reaction as catalyst $\mathbf{D}$ led to the desired product with $63 \%$ ee after $12 \mathrm{~h}$ (Table 1, entry 6). Other secondary free amines like $\mathbf{E}$ and $\mathbf{F}$ displayed similar reactivities at room temperature in DCM (Table 1, entries 7 and 8). Lowering the temperature to $0{ }^{\circ} \mathrm{C}$ increased the enantioselectivity to $87 \%$ using catalyst D (Table 1 , entry 9). At this temperature, further solvents were screened and we found that the enantioselectivity was similar in most tested solvents, with exception of acetonitrile which afforded the product with $47 \%$ ee (Table 1, entries 11-15). However the yield of the reaction was higher in toluene compared to both aprotic and protic polar solvents. Most probably in polar solvents the decomposition of quinoline acetal to quinoline is also going along with the reaction. We also studied the effect of different carbamate protecting groups on nitrogen (see ESI, $\dagger$ for further details). When i-butyl carbamate derivative was used instead of the ethyl carbamate derivative, the corresponding product $\mathbf{3 b}$ was isolated in $72 \%$ yield and $93 \%$ enantiomeric excess (Table 1, entry 16). Next, the generality of the dual catalytic system was studied using propionaldehyde and different quinoline acetals (Table 2).

Both electron-donating and electron-withdrawing groups on the quinoline unit were compatible and furnished the corresponding dihydroquinoline derivatives $\mathbf{3 a - 3 k}$ with high selectivities (90-97\% ee). Use of 6-methyl derived quinoline acetals having different protecting groups on nitrogen illustrated that i-butyl group was slightly superior in this case in terms of both yield and enantioselectivity (Table 2, entries $8 v s$. 7). The reaction was later extended to aldehydes other than propionaldehyde using quinoline acetal 1a. As it can be seen from Table 3, different unfunctionalized aldehydes 2 varying in chain length were well tolerated to give the corresponding products in good yields and selectivities (86-95\% ee). Under the originally developed conditions for propionaldehyde, butyraldehyde gave the product with only $71 \%$ ee (Table 3 , entry 2 ). After screening different parameters like temperature, concentration and catalyst loading, we found that decreasing the temperature to $-30{ }^{\circ} \mathrm{C}$ in toluene increases the enantioselectivity to $89 \%$ although the reaction

Table 2 Scope of the reaction using different quinoline acetals

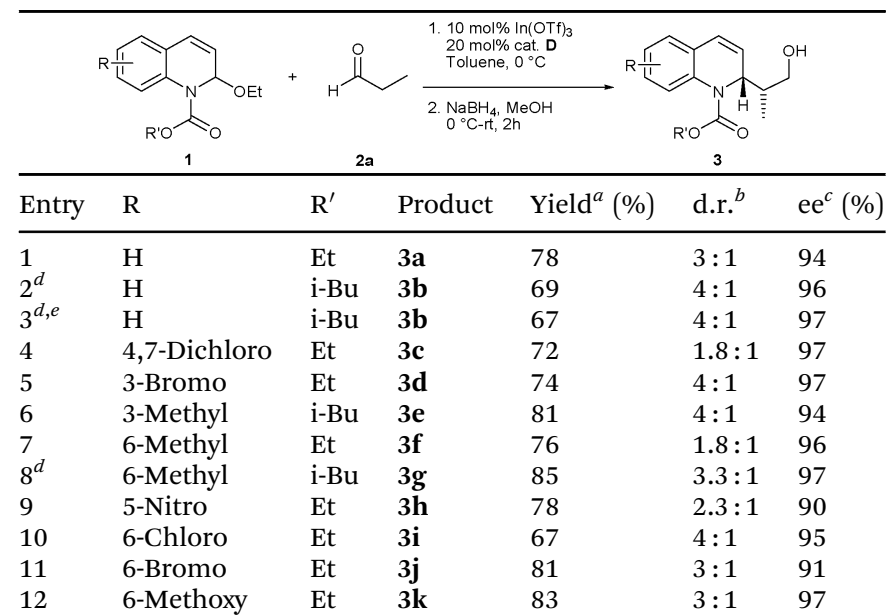

Conditions: 1.0 equiv. of quinolinium acetal and 2.0 equiv. of propionaldehyde in $1.0 \mathrm{~mL}$ of solvent for $18 \mathrm{~h} .{ }^{a}$ Yield after column chromatography. ${ }^{b}$ Diastereomeric ratio was determined by ${ }^{1} \mathrm{H}$-NMR. ${ }^{c}$ Enantiomeric excess of the major diastereomer was determined by chiral HPLC analysis. The enantiomeric excess of the minor diastereomer is given in the ESI. ${ }^{d}$ Reaction was performed at $-10{ }^{\circ} \mathrm{C}$ instead of $0{ }^{\circ} \mathrm{C}$ for $24 \mathrm{~h}$. ${ }^{e} 10 \mathrm{~mol} \%$ of $\mathrm{In}(\mathrm{OTf})_{3}$ and $10 \mathrm{~mol} \%$ of catalyst $\mathbf{D}$. 
Table 3 Scope of the reaction using different aldehydes

\begin{tabular}{|c|c|c|c|c|c|}
\hline & Eto & ${ }_{2}$ & $\begin{array}{l}\text { mol\% } \ln (\mathrm{OTf})_{3} \\
\text { mol\% cat. D } \\
\text { ene, }-30{ }^{\circ} \mathrm{C} \\
\mathrm{BH}_{4,}, \mathrm{MeOH} \\
\mathrm{C}-\mathrm{rt}, 2 \mathrm{~h}\end{array}$ & 3 & \\
\hline Entry & $\mathrm{R}$ & Product & Yield $^{a}(\%)$ & d.r. ${ }^{b}$ & $\mathrm{ee}^{c}(\%)$ \\
\hline $1^{d}$ & $\mathrm{Me}$ & $3 a$ & 78 & $3: 1$ & 94 \\
\hline $2^{d}$ & Et & 31 & 73 & $1.5: 1$ & 71 \\
\hline 3 & Et & 31 & 78 & $1.8: 1$ & 89 \\
\hline 4 & $n$-Pr & $3 \mathrm{~m}$ & 79 & $2.5: 1$ & 95 \\
\hline $5^{e}$ & $n-\operatorname{Pr}$ & $3 n$ & 71 & $3: 1$ & 90 \\
\hline 6 & $n-\mathrm{Bu}$ & 30 & 83 & $3: 1$ & 94 \\
\hline 7 & $\mathrm{Bn}$ & $3 p$ & 63 & $1.5: 1$ & 89 \\
\hline 8 & $n$-Hex & $3 q$ & 72 & $1.8: 1$ & 91 \\
\hline 9 & 2-Propenyl & $3 r$ & 81 & $1.8: 1$ & 86 \\
\hline
\end{tabular}

Conditions: 1.0 equiv. of quinolinium acetal and 3.0 equiv. of aldehyde in $1.0 \mathrm{~mL}$ of solvent for $72 \mathrm{~h}$. ${ }^{a}$ Yield after column chromatography. ${ }^{b}$ Diastereomeric ratio was determined by ${ }^{1} \mathrm{H}$-NMR. ${ }^{c}$ Enantiomeric excess of the major diastereomer was determined by chiral HPLC analysis. The enantiomeric excess of the minor diastereomer is given in the ESI. ${ }^{d}$ Reaction was performed at $0{ }^{\circ} \mathrm{C}$ for $24 \mathrm{~h} .{ }^{e}$ The reaction was done using i-butyl derivative instead of ethyl derivative.

requires longer time (Table 3, entry 3). Good enantioselectivities of 95, 94 and 91\% were observed for the reaction of pentanal, hexanal and octanal respectively (Table 3, entries 4, 6 and 8). 3 -Phenylpropanal reacted sluggishly and led to the product $3 \mathbf{p}$ in $63 \%$ yield and $89 \%$ ee.

The absolute configuration of the dihydroquinoline derivatives was determined by X-ray single crystal analysis of product $\mathbf{3 j}$. Accordingly, the configuration at both centers was established as $(R) .{ }^{17}$ The absolute configuration of the products can be explained by a model depicted in Scheme 2. The E-enamine derived from the reaction of aldehyde and secondary amine catalyst reacts with the iminium ion from the less hindered $S i$-face leading to $(R)$ configuration at the center bearing the aldehyde group. The configuration at the other chiral center depends on the attack of enamine with either $S i$ or $R e$ face of the iminium ion. Our proposal is supported by

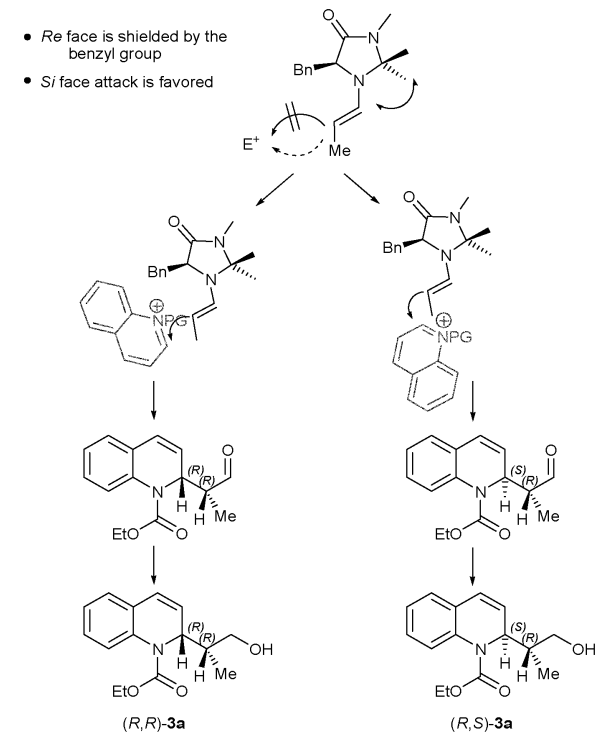

Scheme 2 Model for the addition of enamine onto the iminium ion.

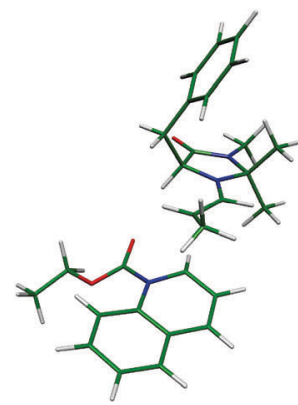

TS1 $(0.0 \mathrm{kcal} / \mathrm{mol})$

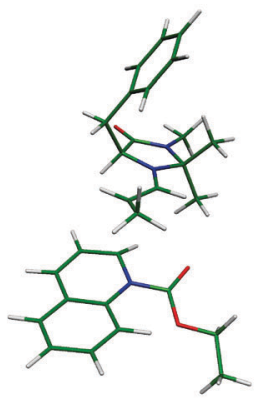

TS2 $(1.29 \mathrm{kcal} / \mathrm{mol})$
Fig. 1 Transition states leading to the $(R, R)$ - and $(R, S)$-3a diastereomers (optimized at the B3LYP/6-31G* level). ${ }^{16}$

theoretical calculations which have shown that the transition state leading to the $(R, R)$-3a diastereomer is by $1.29 \mathrm{kcal} \mathrm{mol}^{-1}$ more favoured as compared to the one leading to the $(R, S)$-3a diastereomer (B3LYP/6-31G* level, Fig. 1). ${ }^{17}$

In order to show the usefulness of our developed protocol, further derivatization of the obtained products was addressed. As shown in Scheme 3, dihydroquinoline product $\mathbf{3 a}$ was reduced to the corresponding tetrahydroquinoline derivative ${ }^{18} 4$ using $5 \mathrm{~mol} \%$ of palladium on charcoal. Next, we attempted the deprotection of carbamate protecting group as this will further illustrate the synthetic potential of the methodology. Treatment of dihydroquinoline alcohol 3a with $\mathrm{KOH}$ in ethanol at $80{ }^{\circ} \mathrm{C}$ gave quinoline 5 in $89 \%$ yield and $92 \%$ ee. ${ }^{19}$ We were delighted to find that under basic conditions the carbamate group was deprotected and the dihydroquinoline was oxidized to afford the quinoline alcohol $\mathbf{5}$ with no significant loss in enantioselectivity. Thus, in this transformation at the end, an asymmetric formal $\alpha$-heteroarylation of aldehydes was realized. The mechanism of this reaction may be either basic hydrolysis of the carbamate group followed by air oxidation to quinolines or $\beta$-elimination of carbonyl group. Both transformations in Scheme 3 were also realized using crude dihydroquinoline alcohol 3a. Next, a diastereoselective hydroetherification could be easily achieved using trimethylsilyl iodide in chloroform at room temperature. Accordingly, quinoline alcohols $\mathbf{3 a}$ and $\mathbf{3} \mathbf{c}$ furnished the corresponding bridged quinoline derivatives 6a and $\mathbf{6 c}$ in high yields and selectivities. 4,7-Dichloroquinoline acetal seems particularly interesting as oxygen attacked the carbon atom containing chlorine atom leading to compound $\mathbf{6 c}$ with a quaternary chiral center (Scheme 4).

In summary, we have developed an effective and dual catalysis protocol in which the combination of a Lewis acid and chiral Lewis base allows the asymmetric addition of aldehydes to quinolinium acetals. The scope of this methodology is very wide as quinoline

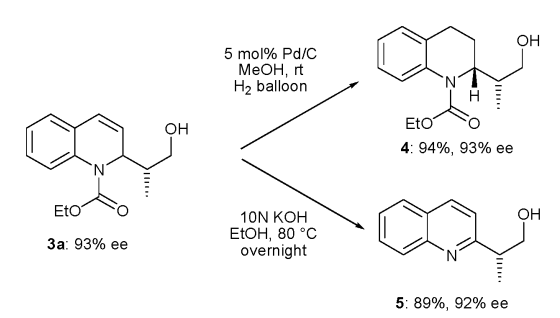

Scheme 3 Functionalization of dihydroquinoline alcohol 3a. 


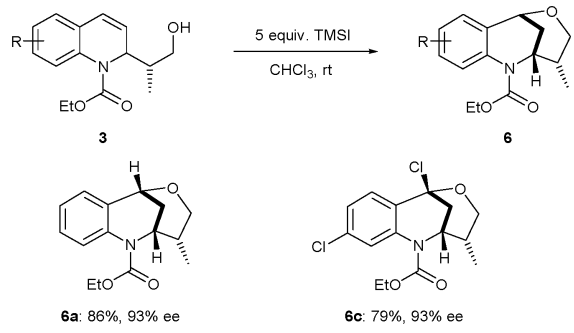

Scheme 4 Lewis acid mediated hydroetherification.

acetals with different substituents are well tolerated under the reaction conditions. ${ }^{20}$ Moreover, the products were easily functionalized under different conditions to allow access to valuable tetrahydroquinolines, 2 -substituted quinolines and bridged quinoline derivatives.

C.M.R.V. gratefully acknowledges the Alexander von Humboldt Foundation (AvH) for a postdoctoral fellowship.

\section{Notes and references}

1 (a) A. R. Katritzky, S. Rachwal and B. Rachwal, Tetrahedron, 1996, 52, 15031-15070; (b) K. M. Witherup, R. W. Ransom, A. C. Graham, A. M. Bernard, M. J. Salvatore, W. C. Lumma, P. S. Anderson, S. M. Pitzenberger and S. L. Varga, J. Am. Chem. Soc., 1995, 117, 6682-6685; (c) R. M. Kariba, P. J. Houghton and A. J. Yenesew, J. Nat. Prod., 2002, 65, 566-569; (d) S. W. Elmore, M. J. Coghlan, D. D. Anderson, J. K. Pratt, B. E. Green, A. X. Wang, M. A. Stashko, C. W. Lin, C. M. Tyree, J. N. Miner, P. B. Jacobsen, D. M. Wilcox and B. C. Lane, J. Med. Chem., 2001, 44, 4481-4491; (e) P. D. Leeson, R. W. Carling, K. W. Morre, A. M. Moseley, J. D. Smith, G. Stevenson, T. Chan, R. Baker and A. C. Foster, J. Med. Chem., 1992, 35, 1954-1968.

2 (a) V. V. Kouznetsov, L. Y. Vargas Méndez and C. M. Meléndez Gómez, Curr. Org. Chem., 2005, 9, 141-161; (b) J. P. Michael, Nat. Prod. Rep., 2007, 24, 223-246.

3 M. Ahamed and M. H. Todd, Eur. J. Org. Chem., 2010, 5935-5942.

4 (a) M. Takamura, K. Funabashi, M. Kanai and M. Shibasaki, J. Am. Chem. Soc., 2000, 122, 6327-6328; (b) M. Takamura, K. Funabashi, M. Kanai and M. Shibasaki, J. Am. Chem. Soc., 2001, 123, 6801-6808. Addition to isoquinolines and pyridines, see: $(c) \mathrm{K}$. Funabashi, H. Ratni, M. Kanai and M. Shibasaki, J. Am. Chem. Soc., 2001, 123, 10784-10785; (d) E. Ichikawa, M. Suzuki, K. Yabu, M. Albert, M. Kanai and M. Shibasaki, J. Am. Chem. Soc., 2004, 126, 11808-11809.

5 Y. Yamaoka, H. Miyabe and Y. Takemoto, J. Am. Chem. Soc., 2007, 129, 6686-6687.

6 Thiourea catalyzed enantioselective addition of enolates to acylisoquinolinium ions, see: M. S. Taylor, N. Tokunaga and E. N. Jacobsen, Angew. Chem., Int. Ed., 2005, 44, 6700-6704.

7 (a) T. J. A. Graham, J. D. Shields and A. G. Doyle, Chem. Sci., 2011, 2, 980-984; (b) J. D. Shields, D. T. Ahneman, T. J. A. Graham and A. G. Doyle, Org. Lett., 2013, 16, 142-145.
8 (a) T. Kodama, P. N. Moquist and S. E. Schaus, Org. Lett., 2011, 13, 6316-6319; for the enantioselective addition of vinyl and aryl boronates to THIQs, see: $(b)$ X. Liu, S. Sun, Z. Meng, H. Lou and L. Liu, Org. Lett., 2015, 17, 2396-2399.

9 (a) S. Mukherjee, J. W. Yang, S. Hoffmann and B. List, Chem. Rev., 2007, 107, 5471-5569; (b) P. Melchiorre, M. Marigo, A. Carlone and G. Bartoli, Angew. Chem., Int. Ed., 2008, 47, 6138-6171; (c) C. F. Barbas III, Angew. Chem., Int. Ed., 2008, 47, 42-47; (d) G. Lelais and D. W. C. MacMillan, Aldrichimica Acta, 2006, 39, 79-87.

10 K. Frisch, A. Landa, S. Saaby and K. A. Jørgensen, Angew. Chem., Int. Ed., 2005, 44, 6058-6063.

11 L. Mengozzi, A. Gualandi and P. G. Cozzi, Chem. Sci., 2014, 5, 3915-3921.

12 J. Zhang, B. Tiwari, C. Xing, X. Chen and Y. R. Chi, Angew. Chem., Int. Ed., 2012, 51, 3649-3652.

13 Organocatalytic asymmetric 1,4-addition of aldehydes to acridinium salts, see: (a) F. Benfatti, E. Benedetto and P. G. Cozzi, Chem. - Asian J., 2010, 5, 2047-2052; (b) T. Liang, J. Xiao, Z. Xiong and X. Li, J. Org. Chem., 2012, 77, 3583-3588.

14 During the preparation of this manuscript similar protocols, were reported: (a) S. Sun, Y. Mao, H. Lou and L. Liu, Chem. Commun., 2015, 51, 10691-10694; (b) F. Berti, F. Malossi, F. Marchetti and M. Pineschi, Chem. Commun., 2015, 51, 13694-13697.

15 Application of $\operatorname{In}(\mathrm{OTf})_{3}$ in combination with imidazolidinone catalysts: (a) R. Sinisi, M. V. Vita, A. Gualamdi, E. Emer and P. G. Cozzi, Chem. - Eur. J., 2011, 17, 7404-7408; (b) M. G. Capdevila, E. Emer, F. Benfatti, A. Gualandi, C. M. Wilson and P. G. Cozzi, Asian J. Org. Chem., 2012, 1, 38-42; (c) A. Gualandi, L. Mengozzi, C. M. Wilson and P. G. Cozzi, Synthesis, 2014, 1321-1328.

16 Examples of combined organocatalysis from our group: (a) M. Rueping, C. M. R. Volla and I. Atodiresei, Org. Lett., 2012, 14, 4642-4645; (b) M. Rueping, R. M. Koenigs, K. Poscharny, D. C. Fabry, D. Leonori and C. Vila, Chem. - Eur. J., 2012, 18, 5170-5174; (c) M. Rueping, J. Dufour and L. Bui, ACS Catal., 2014, 4, 1021-1025; (d) M. Rueping, J. Dufour and M. S. Maji, Chem. Commun., 2012, 48, 3406-3408; (e) M. Rueping and A. P. Antonchick, Angew. Chem., Int. Ed., 2008, 47, 10090-10093; $(f)$ M. Rueping, E. Sugiono and F. R. Schoepke, Synlett, 2007, 1441-1445; $(g)$ M. Rueping, H. Sundén, L. Hubener and E. Sugiono, Chem. Commun., 2012, 48, 2201-2203; (h) M. Rueping, H. Sunden and E. Sugiono, Chem. - Eur. J., 2012, 18, 3649-3653; (i) M. Rueping and R. M. Koenigs, Chem. Commun., 2011, 47, 304-306; $(j)$ M. Rueping and B. N. Nachtsheim, Synlett, 2010, 119-122; $(k)$ M. Rueping, R. M. Koenigs and I. Atodiresei, Chem. - Eur. J., 2010, 16, 9350-9365.

17 See ESI, $\dagger$ for details.

18 V. Sridharan, P. A. Suryavanshi and J. C. Menéndez, Chem. Rev., 2011, 111, 7157-7259.

19 (a) H. Tokuyama, M. Sato, T. Ueda and T. Fukuyama, Heterocycles, 2001, 54, 105-108; $(b)$ T. P. Forrest, G. A. Dauphinee and S. A. Deraniyagala, Can. J. Chem., 1985, 63, 412-417; (c) X. Zhang, T. Yao, M. A. Campo and R. C. Larock, Tetrahedron, 2010, 66, 1177-1187; (d) L. G. Qiang and N. H. Baine, J. Org. Chem., 1988, 53, 4218-4222.

20 Organocatalysis in the synthesis of natural products: (a) E. MarquesLopez, R. P. Herrera and M. Christmann, Nat. Prod. Rep., 2010, 27, 1138-1167; (b) R. M. de Figueiredo and M. Christmann, Eur. J. Org. Chem., 2007, 2575-2600. 